\title{
Cortisol and DHEAS Related to Metabolic Syndrome in Patients with Schizophrenia
}

This article was published in the following Dove Press journal:

Neuropsychiatric Disease and Treatment

\author{
Anastasiia S Boiko (ID \\ Irina A Mednova (D) \\ Elena G Kornetova $\left(^{1,2}\right.$ \\ Nikolay A Bokhan (iD) ${ }^{1,3}$ \\ Arkadiy V Semke $\mathbb{D}^{1}$ \\ Anton JM Loonen (1D ${ }^{4}$ \\ Svetlana A Ivanova (D) ${ }^{1,3}$
}

'Mental Health Research Institute, Tomsk National Research Medical Center of the Russian Academy of Sciences, Tomsk, Russian Federation; ${ }^{2}$ Hospital, Siberian State Medical University, Tomsk, Russian Federation; ${ }^{3}$ Psychiatry, Addictology and Psychotherapy, Siberian State Medical University, Russian Federation;

${ }^{4}$ PharmacoTherapy, -Epidemiology and -Economics, Groningen Research Institute of Pharmacy, University of Groningen, Groningen, The Netherlands

Correspondence: Anton JM Loonen PharmacoTherapy, Epidemiology and Economics, Groningen Research Institute of Pharmacy, University of Groningen, Groningen, The Netherlands

$\mathrm{Tel}+31503637576$

$\mathrm{Fax}+31503632772$

Email a.j.m.loonen@rug.nl
Background: Both dehydroepiandrosterone (DHEAS) and cortisol are secreted by the adrenal glands and may modulate metabolic syndrome (MetS), which often affects the health of patients with schizophrenia. The relationship between the serum levels of these hormones and MetS has not been established.

Purpose: In this pilot study, we investigated the serum levels in schizophrenia patients with and without MetS and compared them with those in healthy volunteers.

Patients and Methods: After obtaining informed consent, 110 patients with acute paranoid schizophrenia were recruited directly after admission to the Mental Health Research Institute. The control group consisted of 51 persons reported on questioning to be mentally and somatically healthy. Blood samples to prepare serum were drawn after an 8-h overnight fast during one of the first days of admission. Serum cortisol and DHEAS concentrations were quantified by enzyme-linked immunosorbent assay.

Results: A total of 42 patients had MetS and 68 patients were without MetS. The cortisol blood level was significantly $(\mathrm{p}=0.012)$ higher in schizophrenia patients without MetS in comparison to healthy controls, while patients with schizophrenia and a MetS have significantly $(p=0.014)$ lower DHEAS levels than healthy volunteers. These differences could, however, exclusively be attributed to female participants. Analysis of covariance adjusted for gender and age demonstrated a significant relationship between age and DHEAS levels $(F=9.512, p=0.003)$.

Conclusion: Lower DHEAS serum levels in relationship to MetS become evident in women, but not in men, and have age differences as a confounding factor.

Keywords: schizophrenia, metabolic syndrome, acute relapse, cortisol, dehydroepiandrosterone, female

\section{Introduction}

Schizophrenia is a major severe mental illness (SMI) with a median lifetime prevalence of 6.35 per 1000 persons (25\% quartile: $4.10 ; 75 \%$ quartile: 8.72$){ }^{1}$ The disease is associated with loss of life expectancy of on average 14.5 (95\% CI 11.2-17.8) years. ${ }^{2}$ This is to an important extent caused by cardiovascular disease, which is associated with schizophrenia with a relative risk of 1.53 (CI95\% $1.27-$ 1.86) in comparison to the incidence in a reference group. ${ }^{3}$ A major contributor to this increased cardiovascular disease burden may be the higher prevalence of metabolic syndrome (MetS), which amounted to an overall rate of $32.5 \%$ (95\% CI $30.1-35.0 \%$ ) in patients with schizophrenia. ${ }^{4}$ In the general population, a diagnosis of MetS has a relative risk of twofold for cardiovascular disease over 5-10 years and at least a fivefold risk for type 2 diabetes. ${ }^{5}$ MetS is characterized by abdominal obesity, hyperglycaemia, dyslipidaemia and hypertension. ${ }^{5}$ MetS has 
been associated with the usage of clozapine and secondgeneration antipsychotics, ${ }^{4,6-8}$ but certain components are also more prevalent in drug-naïve patients with schizophrenia. ${ }^{4}$ Over $20 \%$ of the treatment-naïve schizophrenia patients and first-episode patients were shown to have a lowered HDL-cholesterol level and a glucose dysregulation/insulin resistance. ${ }^{9,10}$ It has been suggested that the effects of inflammatory mediators causally contribute to the pathology of schizophrenia and diabetes that accompanies the disorder. ${ }^{11,12}$ Several studies had delivered evidence for specific cytokine alterations in different stages of schizophrenia. ${ }^{13}$ In a previous paper, we have suggested that certain apolipoproteins (Apos), more specifically ApoA1, may play a role in the pathogenesis of schizophrenia. ${ }^{14}$ ApoA1 and ApoE are also known to have both pro- and anti-inflammatory effects depending on particular circumstances. ${ }^{15-17}$ The same is true for glucocorticoids, ${ }^{18}$ which modulate gene expression by binding to a member of the nuclear receptor family, which affects the expression of pro- and antiinflammatory modulators by immune cells, ${ }^{18-20}$ next to a variety of other cellular effects. ${ }^{19}$ Apart from antiinflammatory activity glucocorticoids also have profound effects on metabolism, which include causing central obesity, hepatic steatosis, insulin resistance, glucose intolerance and dyslipidaemia. ${ }^{21,22}$ Due to their influence on liver, skeletal muscle and adipose tissue as well as on pancreatic alpha and beta cells, chronic exposure to increased levels of circulating cortisol may hence contribute to acquiring MetS via another mechanism than modulating inflammation. ${ }^{22}$ In a meta-analysis of the basal serum cortisol levels of 2953 persons with MetS and 8855 without MetS no significant differences were observed. $^{23}$ This is not necessarily the same in persons with schizophrenia. In spite of substantial heterogeneity, a meta-analysis of 44 studies showed a small-to-medium increase in morning cortisol levels in patients with schizophrenia $(\mathrm{N}=1520)$ compared to controls $(\mathrm{N}=1093) .{ }^{24}$ The same was true for 959 individuals with first-episode psychosis in comparison to 1121 controls included in 27 studies. $^{25}$ Blood cortisol levels were significantly increased in individuals with first-episode psychosis compared to controls with a small-to-medium effect size and significant heterogeneity between the results of the meta-analysed studies. Fasting plasma cortisol was not associated with obesity measures including BMI, waist circumference and visceral fat in 81 subjects with early psychosis. $^{26}$
Dehydroepiandrosterone (DHEA) and its sulfate ester (DHEAS) also regulate the production of pro-inflammatory cytokines, ${ }^{27}$ next to a wide variety of other effects. ${ }^{27-29}$ DHEA(S) is converted into androgens and/or estrogens ${ }^{28}$ and it exerts anti-glucocorticoid activity in adipose tissue ${ }^{29,30}$ and brain. ${ }^{27}$ Both cross-sectional and longitudinal studies have shown that the levels of testosterone may modulate the risk of MetS and diabetes. ${ }^{31}$ However, DHEA(S) regulates adipose tissue metabolism (also) via another mechanism than testosterone. $^{32,33}$ In contrast to testosterone, non-fasting DHEAS serum levels were not related to incident MetS in a prospective cohort of 1004 male adults during a median follow-up time of 5.0 years. ${ }^{34}$ The cortisol/DHEAS ratio has been found to be more associated with MetS in 4255 male Vietnam veterans ${ }^{35}$ and to better predict survival in a group of 313 aged ( $>70$ years old) persons of both genders during an 8 -year follow-up. ${ }^{36}$ In the only study of fasting cortisol/ DHEAS ratio in patients with euthymic bipolar disorder $(\mathrm{N}$ $=60)$ or schizophrenia $(\mathrm{N}=62)$, no significant associations of baseline cortisol levels, DHEAS levels and the cortisol/ DHEAS ratio with the presence of MetS $(n=43)$ were found. ${ }^{37}$ These authors not differentiated between men and women.

The aim of the current pilot study is to determine the possible association between levels of cortisol, DHEAS, their ratio and the presence of MetS in patients with schizophrenia. We hypothesized that cortisol might decrease and DHEAS increases the prevalence of MetS.

\section{Patients and Methods}

\section{Patients}

This study was carried out in accordance with the Code of Ethics of the World Medical Association (Declaration of Helsinki 1975, revised in Fortaleza, Brazil, 2013), established for experiments involving humans. We recruited newly admitted patients from the Mental Health Research Institute Inpatient Department, clinics of Siberian State Medical University and Psychiatric Hospital located in Tomsk, Tomsk oblast (region) of Siberia, Russia. Recruitment started after the study was approved (protocol N187, 24.04.2018) by the Local Bioethics Committee of the Mental Health Research Institute. Each patient provided written informed consent after a proper explanation was given. The inclusion criteria, criteria for MetS and clinical assessments are described in a previous paper. ${ }^{14}$ We included 110 patients with the diagnosis of paranoid schizophrenia verified according to the International Statistical Classification of Diseases, 10th Revision (ICD-10: 
F20) and who were suffering from an acute relapse. Patients have been divided into groups according to criteria of the International Diabetes Federation (IDF, 2005): 42 patients with MetS and 68 patients without MetS. All patients received antipsychotic treatment but may have been incompliant in the previous period.

\section{Control Group}

The control group consisted of 51 persons who were recruited from a group of blood donors, hospital staff members, and students, on a voluntary basis. They reported on questioning to be mentally and somatically healthy individuals.

\section{Blood Sampling}

Laboratory examination was conducted during the first days of hospitalization. Blood samples were drawn after an 8-h overnight fast into tubes with a clot activator $\left(\mathrm{SiO}_{2}\right)$ to isolate the serum (BD Vacutainer). Blood samples with $\mathrm{SiO}_{2}$ were centrifuged for $30 \mathrm{~min}$ at $2000 \times \mathrm{g}$ at $4^{\circ} \mathrm{C}$ to isolate the serum; the serum was stored at $-20^{\circ} \mathrm{C}$ (or $-80^{\circ} \mathrm{C}$ ) until analysis.

\section{Chemical Analysis}

Reagent kits designed to quantify serum cortisol and DHEAS concentrations by enzyme-linked immunosorbent assay (ELISA) were used. The reaction was carried out in accordance with the instructions attached to the kits with the mandatory control of standard serum included in the test system. ELISA results were evaluated on an automatic microplate spectrophotometer at a wavelength of $450 \mathrm{~nm}$. The final results were expressed in units recommended by manufacturers for constructing calibration plots (cortisol in $\mathrm{nmol} / \mathrm{L}$, DHEA-S in $\mu \mathrm{g} / \mathrm{mL}$ ). The units of DHEAS in $\mu \mathrm{g} /$ $\mathrm{mL}$ were converted to $\mathrm{nmol} / \mathrm{L}$ to calculate the Cortisol/ DHEAS ratio. According to the instructions for the ELISA kit, the concentration of the neurosteroid (in $\mu \mathrm{g} / \mathrm{mL}$ ) must be multiplied by a factor of 2700 .

\section{Statistics}

Statistical analyses were performed using the SPSS software for Windows, version 20.0. The data were tested for the normality of the distribution by the Shapiro-Wilk test. Between two-group differences were evaluated using the Mann-Whitney $U$-test for independent samples with nonnormal distribution and Student's $t$-test for samples with normal distribution. Between three-group differences were evaluated using the analysis of variance (ANOVA). Also, a three-group and two-group comparison were carried out by an analysis of covariance (ANCOVA) with gender and age as a covariate. Results were expressed as median with 25\%- and 75\%-quartiles (Me [Q1; Q3]) and mean with standard deviation $(\mathrm{M} \pm \mathrm{SD})$. P-values less than 0.05 were considered as significant.

\section{Results}

Forty-two patients met IDF-criteria for MetS. The characteristics of the patients are specified in Table 1. There were no group differences in gender and clinical (leading symptoms, duration of illness) parameters except for age (patients with MetS were statistically older). Fifty-one healthy individuals $(M / F=13 / 38)$ were recruited to serve as controls.

Table I Socio-Demographic Characteristics of Patients with Schizophrenia and Healthy Individuals

\begin{tabular}{|c|c|c|c|c|}
\hline & Patients without MetS $(n=68)$ & Patients with MetS $(n=42)$ & Healthy Persons $(n=5 I)$ & p-value \\
\hline Age, years $(M \pm S D)$ & $33.93 \pm 8.77$ & $42.42 \pm 11.17$ & $33.21 \pm 9.112$ & $<0.00 I^{*}$ \\
\hline \multicolumn{5}{|l|}{ Male } \\
\hline Quantity, \% (n) & $44.9 \%(3 \mathrm{I})$ & $54.8 \%(23)$ & $25.2 \%(13)$ & \\
\hline Age, years $(M \pm S D)$ & $31.68 \pm 7.071$ & $37.15 \pm 10.456$ & $30.56 \pm 9.948$ & $0.024 *$ \\
\hline \multicolumn{5}{|l|}{ Female } \\
\hline Quantity, \% (n) & $55.1 \%(37)$ & $45.2 \%(19)$ & $74.5 \%(38)$ & \\
\hline Age, years $(M \pm S D)$ & $35.51 \pm 9.009$ & $47.16 \pm 10.777$ & $32.68 \pm 8.388$ & $<0.001 *$ \\
\hline Age of manifestation, years $(M \pm S D)$ & $23.08 \pm 6.271$ & $24.65 \pm 6.765$ & - & 0.07 \\
\hline Duration of illness, years $(M \pm S D)$ & $12.41 \pm 14.559$ & $17.43 \pm 9.363$ & - & $<0.001 *$ \\
\hline PANSS scale, total score $(M \pm S D)$ & $94.73 \pm 17.478$ & $98.15 \pm 15.236$ & - & 0.381 \\
\hline
\end{tabular}

Notes: Comparisons between groups were performed using ANOVA ( 3 groups) and $t$-test ( 2 groups). ${ }^{*} p<0.05-$ statistically significant difference. Abbreviations: MetS, metabolic syndrome; M, mean; SD, standard deviation; PANSS, Positive and Negative Syndrome Scale. 
The serum cortisol level was significantly $(p=0.012)$ higher in schizophrenia patients without MetS in comparison to healthy controls, while patients with schizophrenia and a MetS had significantly $(p=0.014)$ lower DHEAS levels than healthy volunteers (Table 2). Other differences were not significant.

However, female gender turns out to be of major importance. In men neither multiple (Table 3) nor pairwise (data not shown) comparisons between healthy volunteers, schizophrenia patients without MetS and patients with MetS resulted in any significant differences. However, in women multiple comparison resulted in trends or a significant difference. This was mainly attributable to the relatively high cortisol levels in women without MetS in comparison to healthy controls (pairwise comparison: $p=0.003$ ) and the relative low DHEAS levels of patients with MetS in comparison to healthy controls and patients without MetS (pairwise comparisons: $p=0.001$ resp. 0.017).

A three-group ANCOVA with gender and age as a covariate showed that significantly increased cortisol levels remained in the studied groups $(\mathrm{F}=3.220, \mathrm{p}=0.045)$, but we did not find differences in DHEAS levels $(\mathrm{F}=0.331, \mathrm{p}=$ $0.719)$ and cortisol/DHEAS ratio $(F=0.326, p=0.723)$ anymore (Supplementary Table 1). We found relations between age and DHEAS levels $(\mathrm{F}=19.341, \mathrm{p}=0.0001)$ as well as between age and cortisol/DHEAS ratio $(F=5.973, p=0.017)$.

When comparing groups of schizophrenia patients without MetS and healthy controls with ANCOVA adjusted for gender and age, significantly increased cortisol levels remained $(\mathrm{F}=7.023, \mathrm{p}=0.010)$, but also we found relations between cortisol levels and gender $(F=5.617, p=0.021)$.

Comparing groups of schizophrenia patients with MetS and healthy controls with ANCOVA including gender and age as a covariate, we found relations between age and DHEAS levels $(\mathrm{F}=9.512, \mathrm{p}=0.003)$.

Comparison of the relationships between DHEAS, cortisol and the severity of metabolic syndrome as well as psychotic symptoms led to no significant differences (see Supplementary Tables 2 and 3 ). However, due to the many uncertainties about the reasons for possible relationships, our main conclusions were not affected.

\section{Discussion}

The main objective of this pilot study, which was an intermediate step of studying the role of inflammation in causing MetS, was the partial verification of the findings of Vuksan-Ćusa et al, who measured cortisol and DHEAS in a combined group of 60 patients with bipolar disorder and 62 patients with schizophrenia. ${ }^{37}$ We limited our study population to 110 patients with a diagnosis of paranoid schizophrenia and also studied 51 healthy volunteers as a reference group. Our most important finding is that the relationship of particularly DHEAS and MetS differs between the two genders. In men, only small difference was observed between healthy volunteers and patients with or without MetS. In women, however, cortisol levels

Table 2 The Concentration of Cortisol and DHEAS in the Blood Serum of Healthy Individuals and Patients with

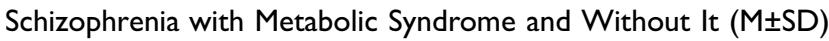

\begin{tabular}{|c|c|c|c|c|}
\hline & Healthy Persons & Patients without MetS & Patients with MetS & p-value \\
\hline Cortisol, $\mathrm{nmol} / \mathrm{L}$ & $522.06 \pm 136.72$ & $621.84 \pm 181.59$ & $578.8 \pm 156.55$ & $\begin{array}{l}P=0.189 \\
P_{1-2}=0.012^{*} \\
P_{1-3}=0.123 \\
P_{2-3}=0.283\end{array}$ \\
\hline DHEAS, $\mu g / \mathrm{mL}$ & $2.92 \pm 1.4$ & $2.53 \pm 1.49$ & $2.1 \pm 1.53$ & $\begin{array}{l}P=0.149 \\
P_{1-2}=0.189 \\
P_{1-3}=0.014 * \\
P_{2-3}=0.148\end{array}$ \\
\hline Cortisol/DHEAS & $0.105 \pm 0.062$ & $0.122 \pm 0.094$ & $0.22 \pm 0.338$ & $\begin{array}{l}\mathrm{P}=0.045^{*} \\
\mathrm{P}_{1-2}=0.39 \mathrm{I} \\
\mathrm{P}_{1-3}=0.063 \\
\mathrm{P}_{2-3}=0.083\end{array}$ \\
\hline
\end{tabular}

Notes: Comparisons between groups were performed using ANOVA and $t$-test. ${ }^{*} \mathrm{p}<0.05$ - statistically significant difference.

Abbreviations: MetS, metabolic syndrome; $M$, mean; SD, standard deviation; DHEAS, dehydroepiandrosterone sulfate; $P_{1-2}$, statistical significance of differences when comparing healthy individuals and patients without MetS; $\mathrm{P}_{1-3}$, statistical significance of differences when comparing healthy individuals and patients with MetS; $\mathrm{P}_{2-3}$, statistical significance of differences when comparing patients with and without MetS. 
Table 3 Cortisol and DHEAS Levels and Arbitrary Cortisol/DHEAS Ratio for Men and Women Separately

\begin{tabular}{|c|c|c|c|c|}
\hline & Healthy Persons & Patients without MetS & Patients with MetS & p-value \\
\hline $\begin{array}{l}\text { Cortisol, nmol/L }(M \pm S D) \\
\text { Male }\end{array}$ & $544.42 \pm 154.91$ & $563.01 \pm 140.89$ & $583.99 \pm 147.68$ & $\begin{array}{l}P=0.884 \\
P_{1-2}=0.722 \\
P_{1-3}=0.462 \\
P_{2-3}=0.657\end{array}$ \\
\hline Female & $528.16 \pm 123.96$ & $669.69 \pm 197.24$ & $572.25 \pm 172.19$ & $\begin{array}{l}\mathrm{P}=0.05 \mathrm{I} \\
\mathrm{P}_{1-2}=0.003^{*} \\
\mathrm{P}_{1-3}=0.338 \\
\mathrm{P}_{2-3}=0.13\end{array}$ \\
\hline $\begin{array}{l}\text { DHEAS, } \mu \mathrm{g} / \mathrm{mL}(\mathrm{M}[\mathrm{Q} \mathrm{I} ; \mathrm{Q} 3]) \\
\text { Male }\end{array}$ & $2.662[1.969 ; 3.235]$ & $2.680[1.775 ; 3.929]$ & $2.243[1.331 ; 3.225]$ & $\begin{array}{l}P=0.38 \\
P_{1-2}=0.709 \\
P_{1-3}=0.673 \\
P_{2-3}=0.49\end{array}$ \\
\hline Female & $2.264[1.520 ; 3.307]$ & $1.956[1.166 ; 2.860]$ & 1.187 [0.953; 1.998$]$ & $\begin{array}{l}P=0.092 \\
P_{1-2}=0.191 \\
P_{1-3}=0.001 * \\
P_{2-3}=0.017^{*}\end{array}$ \\
\hline $\begin{array}{l}\text { Cortisol/DHEAS }(M \pm S D) \\
\text { Male }\end{array}$ & $0.084 \pm 0.019$ & $0.099 \pm 0.078$ & $0.23 \pm 0.42$ & $\begin{array}{l}P=0.752 \\
P_{1-2}=0.568 \\
P_{1-3}=0.313 \\
P_{2-3}=0.204\end{array}$ \\
\hline Female & $0.114 \pm 0.07$ & $0.142 \pm 0.103$ & $0.208 \pm 0.197$ & $\begin{array}{l}P=0.003^{*} \\
P_{1-2}=0.308 \\
P_{1-3}=0.043 * \\
P_{2-3}=0.188\end{array}$ \\
\hline
\end{tabular}

Notes: Comparisons between groups were performed using ANOVA, $t$-test and Mann-Whitney U-test. ${ }^{*} p<0.05-$ statistically significant difference.

Abbreviations: MetS, metabolic syndrome; M, mean/median; SD, standard deviation; QI, lower quartile; Q3, upper quartile; DHEAS, dehydroepiandrosterone sulfate; $\mathrm{P}_{\mathrm{I}-2}$, statistical significance of differences when comparing healthy individuals and patients without MetS; $\mathrm{P}_{\mathrm{I}-3}$, statistical significance of differences when comparing healthy individuals and patients with MetS; $P_{2-3}$, statistical significance of differences when comparing patients with and without MetS.

tend to be higher in patients in comparison to healthy individuals, but DHEAS levels are lower and particularly so in patients with MetS. However, the latter relationship is confounded by age differences. In their study, VuksanĆusa et al did not discriminate between men and women.

The interpretation of our results is not a simple matter. We actually did not obtain support for a possible association between cortisol levels and MetS. However, in women, but hardly not in men, cortisol levels were found to be higher in persons with schizophrenia. This partly corresponds to the results of the published meta-analysis. ${ }^{24,25}$ However, a direct comparison between male and female patients versus controls was absent from these studies. Two-group ANCOVA adjusted for gender and age still demonstrated increased cortisol levels in schizophrenia patients without MetS and also the relationship between cortisol levels and gender. In our population,
DHEAS levels were lowest in patients with MetS. In females, a pairwise comparison with patients without MetS as well as healthy volunteers resulted in a significant difference. DHEA is known to have insulin-promoting effects by enhancing insulin release and insulin receptor sensitivity. ${ }^{32,33}$ However, non-fasting DHEAS serum levels were not related to incident MetS in a prospective cohort of 1004 male adults during a median follow-up time of 5.0 years. ${ }^{34}$ In a meta-analysis of 13 studies, Misiak et al observed significantly higher levels of DHEAS in a group of patients with schizophrenia compared to controls in a pooled analysis of all studies. ${ }^{38}$ However, subgroup analysis revealed that no significant differences were found in acutely relapsed (3 studies) and stable (5 studies) multiple-episode patients. Our findings concerning female patients with schizophrenia versus controls differ from those of Bulut et al, who compared 32 pre-menopausal 
women with stable schizophrenia and 32 age- and sexmatched healthy controls. ${ }^{39}$ However, although several investigators report higher levels in female patients versus controls, ${ }^{40}$ other researchers obtained contrasting results. ${ }^{41,42}$ The most important difference within the context of gender aspects of DHEA-physiology is, of course, the high levels of testosterone in males. In men, gonadal testosterone is most important, while in women the major source of androgens is DHEAS. $^{43} \mathrm{DHEA}(\mathrm{S})$ is converted into testosterone and estradiol, and in individuals with panhypopituitarism, whose gonads do not produce sex hormones, a $50 \mathrm{mg}$ dose of DHEA resulted in DHEA plasma levels as observed in young adults, in testosterone levels similar to those observed in young women and estradiol levels were within the normal range observed in men. ${ }^{44}$ The problem with DHEA is that DHEA secretion peaks at age 25-30 yr and declines thereafter. $^{43,44}$ The results of ANCOVA adjusted for gender and age demonstrated a confounding influence of age difference in our study. Age differences may contribute to the lower DHEAS levels we found in female patients in comparison to males, but we cannot be certain that this sufficiently explains all differences. Aged-matched, longitudinal studies are probably necessary to investigate how the disease process, mensis, menopause and pharmacological effects modify DHEAS production during the course of time and to elucidate the background of this difference between female patients and control subjects. Perhaps, higher testosterone levels in males prevent that the effects of a DHEAS-related factor, whatever that may be, become evident. A possibility might be that higher metabolism of DHEAS in patients with MetS is inhibited by testosterone. Therefore, parallel measurements of pituitary (ACTH, FSH, prolactin), adrenal (cortisol, DHEAS), gonadal (testosterone, estradiol, progesterone), and metabolic hormones (insulin, ghrelin, leptin) of age-matched patients with and without metabolic syndrome, in women in relationship to the menstrual cycle and menopause, are indicated for the future.

This pilot study has several limitations. We measured only cortisol and DHEAS and the tested individuals were not completely aged-matched with each other. Usually, it is difficult while doing this type of research to find sufficiently healthy persons of comparable age and sex to the studied patient group. Many female patients with schizophrenia have secondary amenorrhoea or (like most young women) use contraceptive drugs. When a cohort of patients with schizophrenia is divided into persons with or without MetS, the first group has typically an older age. The findings of this study are preliminary in a certain respect and need verification, but it may be useful to distinguish male and female patients when studying the mechanism of metabolic syndrome.

\section{Conclusion}

We measured cortisol and dehydroepiandrosterone sulphate (DHEAS) serum levels in 51 healthy individuals and 110 patients with paranoid schizophrenia of which 42 had a metabolic syndrome (MetS) and 68 patients not. Our results indicate that female gender turns out to be of major importance. In men, no statistically significant differences between the three groups were observed. In women, relatively high cortisol levels were found in women without MetS in comparison to healthy controls and relatively low DHEAS levels in patients with MetS in comparison to healthy controls and patients without MetS.

\section{Acknowledgments}

The authors are grateful to the Siberian State Medical University for their help in recruiting healthy volunteers and patients.

\section{Funding}

This work was supported by the Russian Science Foundation, grant \# 18-15-00011.

\section{Disclosure}

Dr Anastasiia S Boiko reports grants from the Russian Science Foundation, during the conduct of the study. Dr Irina A Mednova reports grants from the Russian Science Foundation, during the conduct of the study. Dr Elena G Kornetova reports grants from the Russian Science Foundation, during the conduct of the study. Prof. Dr Svetlana A Ivanova reports grants from the Russian Science Foundation, during the conduct of the study; nonfinancial support, receives salary as a professor from the Siberian State Medical University, outside the submitted work; The authors declare that this research was conducted in the absence of any commercial or financial relationships that could be considered as a potential conflict of interest.

\section{References}

1. Moreno-Kustner B,C, Martin C, Pastor L, McKenna PJ. Prevalence of psychotic disorders and its association with methodological issues. A systematic review and meta-analyses. PLoS One. 2018;13(4): e0195687. doi:10.1371/journal.pone.0195687

2. Hjorthoj C, Sturup AE, McGrath JJ, Nordentoft M. Years of potential life lost and life expectancy in schizophrenia: a systematic review and meta-analysis. Lancet Psychiatry. 2017;4(4):295-301. doi:10.1016/ S2215-0366(17)30078-0 
3. Fan Z, Wu Y, Shen J, Ji T, Zhan R. Schizophrenia and the risk of cardiovascular diseases: a meta-analysis of thirteen cohort studies. $J$ Psychiatr Res. 2013;47(11):1549-1556. doi:10.1016/j.jpsychires. 2013.07.011

4. Mitchell AJ, Vancampfort D, Sweers K, van Winkel R, Yu W, De Hert M. Prevalence of metabolic syndrome and metabolic abnormalities in schizophrenia and related disorders-a systematic review and meta-analysis. Schizophr Bull. 2013;39(2):306-318. doi:10.1093/ schbul/sbr148

5. Samson SL, Garber AJ. Metabolic syndrome. Endocrinol Metab Clin North Am. 2014;43(1):1-23. doi:10.1016/j.ec1.2013.09.009

6. Bou Khalil R. Atypical antipsychotic drugs, schizophrenia, and metabolic syndrome in Non-Euro-American societies. Clin Neuropharmacol. 2012;35(3):141-147. doi:10.1097/WNF.0b013e31824d5288

7. Hirsch L, Yang J, Bresee L, Jette N, Patten S, Pringsheim T. Secondgeneration antipsychotics and metabolic side effects: A systematic review of population-based studies. Drug Saf. 2017;40(9):771-781. doi:10.1007/s40264-017-0543-0

8. Vancampfort D, Stubbs B, Mitchell AJ, et al. Risk of metabolic syndrome and its components in people with schizophrenia and related psychotic disorders, bipolar disorder and major depressive disorder: a systematic review and meta-analysis. World Psychiatry. 2015;14(3):339-347. doi:10.1002/wps.20252

9. Mitchell AJ, Vancampfort D, De Herdt A, Yu W, De Hert M. Is the prevalence of metabolic syndrome and metabolic abnormalities increased in early schizophrenia? A comparative meta-analysis of first episode, untreated and treated patients. Schizophr Bull. 2013;39 (2):295-305. doi:10.1093/schbul/sbs082

10. Anjum S, Bathla M, Panchal S, Singh GP, Singh M. Metabolic syndrome in drug naïve schizophrenic patients. Diabetes Metab Syndr. 2018;12(2):135-140. doi:10.1016/j.dsx.2017.11.006

11. Leonard BE, Schwarz M, Myint AM. The metabolic syndrome in schizophrenia: is inflammation a contributing cause? J Psychopharmacol. 2012;26(5_suppl):33-41. doi:10.1177/0269881 111431622

12. Hoffman RP. The complex inter-relationship between diabetes and Schizophrenia. Curr Diabetes Rev. 2017;13(3):528-532. doi:10.2174/ 1573399812666161201205322

13. Upthegrove R, Khandaker GM. Cytokines, oxidative stress and cellular markers of inflammation in schizophrenia. Curr Top Behav Neurosci. 2020;44:49-66.

14. Boiko AS, Mednova IA, Kornetova EG, et al. Apolipoprotein serum levels related to metabolic syndrome in patients with schizophrenia. Heliyon. 2019;5(7):e02033. doi:10.1016/j.heliyon.2019.e02033

15. Navab M, Ananthramaiah GM, Reddy ST, et al. The double jeopardy of HDL. Ann Med. 2005;37(3):173-178. doi:10.1080/078538905 10007322

16. Namiri-Kalantari R, Gao F, Chattopadhyay A, et al. The dual nature of HDL: anti-inflammatory and pro-inflammatory. Biofactors. 2015;41(3):153-159. doi:10.1002/biof.1205

17. Guo L, LaDu MJ, Van Eldik LJ. A dual role for apolipoprotein e in neuroinflammation: anti- and pro-inflammatory activity. $J$ Mol Neurosci. 2004;23(3):205-212. doi:10.1385/JMN:23:3:205

18. Desmet SJ, De Bosscher K. Glucocorticoid receptors: finding the middle ground. J Clin Invest. 2017;127(4):1136-1145. doi:10.1172/ JCI88886

19. Baschant U, Tuckermann J. The role of the glucocorticoid receptor in inflammation and immunity. J Steroid Biochem Mol Biol. 2010;120 (2-3):69-75. doi:10.1016/j.jsbmb.2010.03.058

20. Oakley RH, Cidlowski JA. The biology of the glucocorticoid receptor: new signaling mechanisms in health and disease. J Allergy Clin Immunol. 2013;132(5):1033-1044. doi:10.1016/j.jaci.2013.09.007

21. van Raalte DH, Ouwens DM, Diamant M. Novel insights into glucocorticoid-mediated diabetogenic effects: towards expansion of therapeutic options? Eur J Clin Invest. 2009;39(2):81-93. doi:10.1 111/j.1365-2362.2008.02067.x
22. van Raalte DH, Diamant M. Steroid diabetes: from mechanism to treatment? Neth J Med. 2014;72(2):62-72.

23. Garcez A, Leite HM, Weiderpass E, et al. Basal cortisol levels and metabolic syndrome: A systematic review and meta-analysis of observational studies. Psychoneuroendocrinology. 2018;95:50-62. doi:10.1016/j.psyneuen.2018.05.023

24. Girshkin L, Matheson SL, Shepherd AM, Green MJ. Morning cortisol levels in schizophrenia and bipolar disorder: a meta-analysis. Psychoneuroendocrinology. 2014;49:187-206. doi:10.1016/j.psyneuen. 2014.07.013

25. Hubbard DB, Miller BJ. Meta-analysis of blood cortisol levels in individuals with first-episode psychosis. Psychoneuroendocrinology. 2019;104:269-275. doi:10.1016/j.psyneuen.2019.03.014

26. Manzanares N, Monseny R, Ortega L, et al. Unhealthy lifestyle in early psychoses: the role of life stress and the hypothalamic-pituitaryadrenal axis. Psychoneuroendocrinology. 2014;39:1-10. doi:10.1016/ j.psyneuen.2013.09.023

27. Maninger N, Wolkowitz OM, Reus VI, Epel ES, Mellon SH. Neurobiological and neuropsychiatric effects of dehydroepiandrosterone (DHEA) and DHEA sulfate (DHEAS). Front Neuroendocrinol. 2009;30(1):65-91. doi:10.1016/j.yfrne.2008.11.002

28. Labrie F, Luu-The V, Bélanger A, et al. Is dehydroepiandrosterone a hormone? J Endocrinol. 2005;187(2):169-196. doi:10.1677/joe.1.06264

29. Karbowska J, Kochan Z. Effects of DHEA on metabolic and endocrine functions of adipose tissue. Horm Mol Biol Clin Investig. 2013;14(2):65-74. doi:10.1515/hmbci-2013-0009

30. McNelis JC, Manolopoulos KN, Gathercole LL, et al. Dehydroepiandrosterone exerts antiglucocorticoid action on human preadipocyte proliferation, differentiation, and glucose uptake. $\mathrm{Am}$ J Physiol Endocrinol Metab. 2013;305(9):E1134-1144. doi:10.1152/ ajpendo.00314.2012

31. Kim C, Halter JB. Endogenous sex hormones, metabolic syndrome, and diabetes in men and women. Curr Cardiol Rep. 2014;16(4):467. doi:10.1007/s11886-014-0467-6

32. De Pergola G. The adipose tissue metabolism: role of testosterone and dehydroepiandrosterone. Int J Obes Relat Metab Disord. 2000;24 (Suppl 2):S59-63. doi:10.1038/sj.ijo.0801280

33. Aoki K, Terauchi Y. Effect of dehydroepiandrosterone (DHEA) on diabetes mellitus and obesity. Vitam Horm. 2018;108:355-365.

34. Haring R, Völzke H, Felix SB, et al. Prediction of metabolic syndrome by low serum testosterone levels in men: results from the study of health in Pomerania. Diabetes. 2009;58(9):2027-2031. doi: $10.2337 / \mathrm{db} 09-0031$

35. Phillips AC, Carroll D, Gale CR, Lord JM, Arlt W, Batty GD. Cortisol, DHEAS, their ratio and the metabolic syndrome: evidence from the Vietnam Experience Study. Eur J Endocrinol. 2010;162 (5):919-923. doi:10.1530/EJE-09-1078

36. Mora M, Serra-Prat M, Palomera E, Puig-Domingo M. Metabolic and hormonal contributors to survival in the participants of the Mataró ageing study at 8 years follow-up. Clin Endocrinol (Oxf). 2014;81 (5):775-783. doi:10.1111/cen.12467

37. Vuksan-Ćusa B, Sagud M, Mihaljević-Peleš A, Jakšić N, Jakovljević M. Metabolic syndrome and cortisol/DHEAS ratio in patients with bipolar disorder and schizophrenia. Psychiatr Danub. 2014;26(2):187-189.

38. Misiak B, Frydecka D, Loska O, et al. Testosterone, DHEA and DHEA-S in patients with schizophrenia: A systematic review and meta-analysis. Psychoneuroendocrinology. 2018;89:92-102. doi:10. 1016/j.psyneuen.2018.01.007

39. Bulut SD, Bulut S, Gundogmus AG, Aydemir C. Serum DHEA-S, testosterone and cortisol levels in female patients with schizophrenia. Endocr Metab Immune Disord Drug Targets. 2018;18(4):348-354. doi:10.2174/1871530318666180212102128

40. da Silva TL, Ravindran AV. Contribution of sex hormones to gender differences in schizophrenia: A review. Asian $J$ Psychiatr. 2015;18:2-14. doi:10.1016/j.ajp.2015.07.016 
41. Bičíková M, Hampl R, Hill M, Řípová D, Mohr P, Putz Z. Neuroand immunomodulatory steroids and other biochemical markers in drug-naive schizophrenia patients and the effect of treatment with atypical antipsychotics. Neuro Endocrinol Lett. 2011;32(2):141-147.

42. Huang YC, Hung CF, Lin PY, et al. Gender differences in susceptibility to schizophrenia: potential implication of neurosteroids. Psychoneuroendocrinology. 2017;84:87-93. doi:10.1016/j.psyneuen. 2017.06.017
43. Labrie F, Martel C, Bélanger A, Pelletier G. Androgens in women are essentially made from DHEA in each peripheral tissue according to intracrinology. J Steroid Biochem Mol Biol. 2017;168:9-18. doi:10.1016/j.jsbmb.2016.12.007

44. Young J, Couzinet B, Nahoul K, et al. Panhypopituitarism as a model to study the metabolism of dehydroepiandrosterone (DHEA) in humans. $J$ Clin Endocrinol Metab. 1997;82(8):2578-2585. doi:10.1210/jcem.82.8.4157

\section{Publish your work in this journal}

Neuropsychiatric Disease and Treatment is an international, peerreviewed journal of clinical therapeutics and pharmacology focusing on concise rapid reporting of clinical or pre-clinical studies on a range of neuropsychiatric and neurological disorders. This journal is indexed on PubMed Central, the 'PsycINFO' database and CAS, and is the official journal of The International Neuropsychiatric Association (INA). The manuscript management system is completely online and includes a very quick and fair peer-review system, which is all easy to use. Visit http://www.dovepress.com/testimonials.php to read real quotes from published authors. 